\title{
DESENVOLVIMENTO DE UM SISTEMA DE CONTROLE E AUTOMAÇÃO PARA ESTUDO DO PROCESSO DE LAMINAÇÃO DE METAIS NÃO FERROSOS NO LAMINADOR MACHINE DUO REVERSÍVEL DE 8 TON*
}

\author{
Vantuil Manoel Thebas ${ }^{1}$ \\ Marcelo Lucas Pereira Machado² \\ José Denti Filho ${ }^{3}$
}

\section{Resumo}

A automatização do laminador Machine duo reversível de 8 toneladas passa pela determinação da força de laminação em seus cilindros durante processo de deformação da chapa metálica não ferrosa, o que se faz pela medição direta da força nos cilindros do laminador através de um sensor de força ou pelo uso de modelos matemáticos aplicados ao processo de laminação das ligas metálicas. $O$ presente trabalho tem por objetivo o estudo dos modelos de Orowan, Ekelund e Bland \& Ford aplicados à predição da carga de laminação de ligas metálicas não ferrosas. Amostras em barras chatas extrudadas com $6,35 \mathrm{~mm}\left(1 / 4^{\prime}\right)$ de espessura da liga de Alumínio 6063-T5, com $300 \mathrm{~mm}$ de comprimento e 50,8 mm (2') de largura, foram laminadas. Suas dimensões finais foram obtidas por uma escala de medição e paquímetro universal, sendo os dados tratados por rotinas computacionais especificamente desenvolvidas para a implementação dos modelos matemáticos citados. Os resultados deram conta de que a implementação dos modelos de Orowan, Ekelund e Bland \& Ford através de rotinas computacionais podem ser muito úteis na predição da carga de laminação de ligas metálicas não ferrosas.

Palavras-chave: Laminação; Orowan; Ekelund; Bland \& Ford.

\section{DEVELOPMENT OF A SYSTEM OF CONTROL AND AUTOMATION FOR STUDY OF ROLLING PROCESS OF NON-FERROUS METALS ON THE MILL MACHINE IN REVERSE DUO 8 TON}

\section{Abstract}

Automation of Machine rolling mill reversible duo 8 tons goes by determining the strength of lamination cylinders during the deformation process of nonferrous metal plate, which makes the direct measurement of force in rolls of the rolling mill by a force sensor or by use of mathematical models applied to the rolling process of alloys. The present work aims to study the models Orowan, Ekelund and Bland \& Ford applied to the prediction of rolling load of nonferrous alloys. Samples in extruded flat bars with $6.35 \mathrm{~mm}(1 / 4$ ") thick aluminum alloy $6063-\mathrm{T} 5,300 \mathrm{~mm}$ long and $50.8 \mathrm{~mm}$ (2") wide, were laminated. Its final dimensions were obtained by a measuring scale and universal caliper, and the data processed by computer routines developed specifically for the implementation of the aforementioned mathematical models. The results realized that the implementation of models Orowan, Ekelund and Bland \& Ford through computational routines can be very useful in predicting the load rolling of nonferrous alloys.

Keywords: Lamination; Orowan; Ekelund; Bland \& Ford.

1 Engenheiro Eletricista, Professor, Coordenadoria de Automação Industrial, Instituto Federal do Espírito Santo (IFES), Serra, Espírito Santo, Brasil.

2 Doutor em Engenharia Elétrica, Professor, Curso de Mestrado em Engenharia Metalúrgica e de Materiais, Instituto Federal do Espírito Santo (IFES), Vitória, Espírito Santo, Brasil.

3 Doutor em Engenharia Metalúrgica e de Minas, Professor Associado IV, Universidade Federal do Espírito Santo (UFES), Vitória, Espírito Santo, Brasil.

* Contribuição técnica ao 69 Congresso Anual da ABM - Internacional e ao 14ํㅡㄹ ENEMET - Encontro Nacional de Estudantes de Engenharia Metalúrgica, de Materiais e de Minas, 21 a 25 de julho de 2014, São Paulo, SP, Brasil. 


\section{INTRODUÇÃO}

Segundo Machado [1], a laminação é um processo de conformação mecânica no qual o material é forçado a passar entre dois cilindros, girando em sentidos opostos com a mesma velocidade superficial, separados entre si por uma distância menor que o valor da espessura inicial do material a ser deformado. A automatização do laminador passa pela determinação dessa força de laminação, a qual depende de diversos fatores tais como a temperatura do material a ser laminado, o valor da redução da espessura da amostra no passe, o atrito entre o material laminado e os cilindros, a velocidade de laminação, a composição química da liga metálica, a área de contato entre o material e os cilindros dentre outros. A correta medição dessa força torna-se fundamental em quaisquer estudos de laminação de ligas metálicas, ferrosas e não ferrosas.

A implementação dos modelos de Orowan, Ekelund e Bland \& Ford por rotinas computacionais visando a predição da carga de laminação em ligas metálicas nãoferrosas constitui-se uma das etapas mais importantes da automatização do laminador duo reversível de 8 ton. A implementação de tais rotinas computacionais exige o conhecimento da teoria de laminação, a qual é importante não somente para a realização de novos projetos mas também ajuda o técnico na escolha de programas de otimização adequados através da alteração das condições de trabalho do maquinário industrial.

Uma estimativa para a carga de laminação de chapas a frio pode ser obtida considerando o processo de laminação como um processo de compressão homogênea, ou seja, a distribuição de pressão ao longo do arco de contato é considerada uniforme. A área de contato dos cilindros pode ser determinada pela expressão

Onde:

$$
A=L \cdot W=(\sqrt{R . \Delta h}) \cdot W
$$

A - Área de contato dos cilindros com a chapa laminada, em $\mathrm{mm}^{2}$;

$\mathrm{L}$ - Comprimento do arco de contato, em $\mathrm{mm}$;

W - Largura da chapa laminada, em $\mathrm{mm}$;

$\mathrm{R}$ - Raio dos cilindros do laminador sem deformação elástica, em mm;

$\Delta \mathrm{h}$ - Redução de espessura da chapa, em $\mathrm{mm}$.

\subsection{Modelo de Orowan}

Admitindo-se que não ocorra deformação lateral, ou seja, que hi é muito menor que W, a razão da carga de laminação pela largura da chapa pode ser dada por

Onde:

$$
\frac{P}{W}=\bar{S} \sqrt{R \cdot \Delta h}
$$

$\mathrm{P}$ - Carga de laminação aplicada pelos cilindros à chapa, em Kgf;

W - Largura da chapa laminada, em $\mathrm{mm}$;

$\bar{S}=\frac{2}{\sqrt{3}} \bar{Y}-$ Tensão de escoamento média biaxial, em $\mathrm{Kgf} / \mathrm{mm}^{2}$;

$\bar{Y}$ - Tensão de escoamento média uniaxial, em $\mathrm{Kgf} / \mathrm{mm}^{2}$;

$\mathrm{R}$ - Raio dos cilindros do laminador sem deformação elástica, em mm;

$\Delta \mathrm{h}$ - Redução de espessura da chapa, em $\mathrm{mm}$.

Segundo Helman et al. [2], esta expressão fornece um valor sempre inferior ao real por não considerar o efeito do atrito, devendo ser acrescida em $20 \%$ do valor da

* Contribuição técnica ao 69 Congresso Anual da ABM - Internacional e ao 14ํㅡㄹ ENEMET - Encontro Nacional de Estudantes de Engenharia Metalúrgica, de Materiais e de Minas, 21 a 25 de julho de 2014, São Paulo, SP, Brasil. 


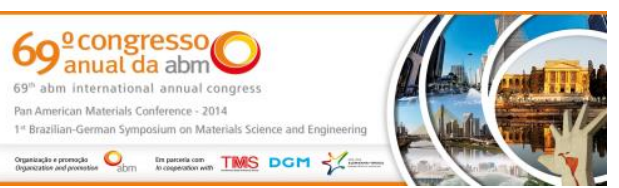

carga para compensar esse efeito. Assim, o valor da carga por unidade de largura será dado por

\subsection{O Modelo de Ekelund}

$$
\frac{P}{W}=1,2 \cdot \bar{S} \cdot \sqrt{R \cdot \Delta h}
$$

Outra expressão de grande utilidade para o cálculo da carga de laminação, por sua facilidade matemática e razoável precisão, foi proposta por Ekelund (apud Helman et al. [2]). Nela aparece o raio deformado dos cilindros (R') o qual pode ser calculado através da equação de Hitchcock (apud Ginzburg [3]). O modelo de Ekelund para o cálculo da carga de laminação pode ser expresso por

Onde:

$$
\frac{P}{W}=\bar{S} \sqrt{R^{\prime} \Delta h}\left[1+\frac{1,6 \mu \sqrt{R^{\prime} \Delta h}-1,2 \Delta h}{h_{i}+h_{f}}\right]
$$

$\mathrm{P}$ - Carga de laminação aplicada pelos cilindros à chapa, em Kgf;

W - Largura da chapa laminada, em $\mathrm{mm}$;

$\bar{S}=\frac{2}{\sqrt{3}} \bar{Y}-$ Tensão de escoamento média biaxial, em $\mathrm{Kgf} / \mathrm{mm}^{2}$;

$\bar{Y}$ - Tensão de escoamento média uniaxial, em $\mathrm{Kgf} / \mathrm{mm}^{2}$;

$\mathrm{R}^{\prime}$ - Raio deformado dos cilindros do laminador, em $\mathrm{mm}$;

$\Delta \mathrm{h}$ - Redução de espessura da chapa, em mm;

$\mu$ - Coeficiente de atrito entre chapa e cilindros do laminador, um adimensional;

$h_{i}$ e $h_{f}-$ Espessura inicial e final da chapa respectivamente, em $\mathrm{mm}$.

Helman et al. [2] afirma que com as elevadas pressões desenvolvidas durante a laminação a frio não se pode ignorar a deformação elástica nos cilindros do laminador, uma vez que ela exerce influência marcante sobre a carga de laminação e a espessura mínima da chapa que se pode produzir nesse laminador. Assim, nas expressões que determinam a carga de laminação aparece um valor R' diferente de $\mathrm{R}$ para o raio do arco de contato. Esse valor não leva em consideração a deformação elástica da chapa e assume que a distribuição de pressão no arco de contato é aproximadamente elíptica. O valor R' pode ser calculado pela expressão de Hitchcock (apud Ginzburg [3]), que conduz a resultados satisfatórios num amplo intervalo de espessuras e reduções do material, sendo particularmente adequada para inclusão em programas computacionais para cálculo de sequência de passe.

Onde:

$$
R^{\prime}=R\left[1+\frac{c}{h_{i}-h_{f}}\left(\frac{P}{W}\right)\right]
$$

R' - Raio deformado dos cilindros do laminador, em mm;

$\mathrm{R}$ - Raio dos cilindros do laminador sem deformação elástica, em mm;

$c=\frac{16\left(1-v^{2}\right)}{\pi E}$, onde o parâmetro $v$ (Módulo de Poisson) é um adimensional e o parâmetro $E$ (Módulo de Young) é dado em $\mathrm{Kgf} / \mathrm{mm}^{2}$;

$\mathrm{P}$ - Carga de laminação aplicada pelos cilindros à chapa, em Kgf;

W - Largura da chapa laminada, em $\mathrm{mm}$;

$h_{i}$ e $h_{f}-$ Espessura inicial e final da chapa respectivamente, em $\mathrm{mm}$.

\subsection{O Modelo de Bland \& Ford}

Um modelo fundamentado na teoria da laminação e destinado ao cálculo da carga de laminação a frio foi proposto por Bland \& Ford (apud Helman et al. [2]). Este

* Contribuição técnica ao 69 Congresso Anual da ABM - Internacional e ao 14ํㅡㄹ ENET - Encontro Nacional de Estudantes de Engenharia Metalúrgica, de Materiais e de Minas, 21 a 25 de julho de 2014, São Paulo, SP, Brasil. 


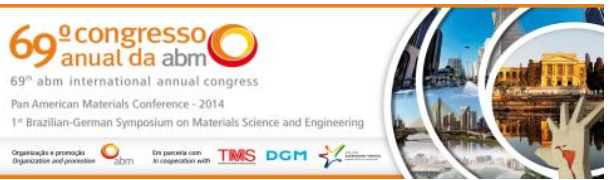

modelo realiza uma análise da distribuição de tensões e deformações locais baseada na geometria do passe, e para isso faz as seguintes suposições:

$i$ - Estado plano de deformações;

ii - Deformação homogênea em cada secção plana;

iii - Coeficiente de atrito constante em cada arco de contato;

iv - Arco de contato circular de raio R';

$v$ - Deformação elástica desprezível da chapa.

$\mathrm{Na}$ figura 1 a seguir nota-se o ponto A na região de saída do arco de contato e também o ponto $B$ na região de entrada, separados pelo ponto $C$, ponto de convergência das forças de atrito da chapa laminada.

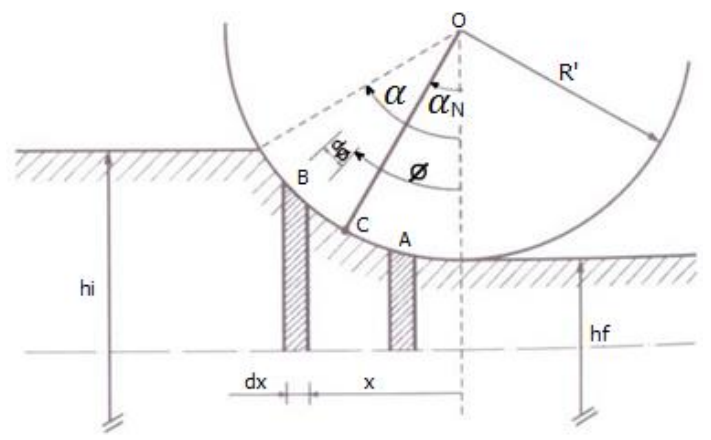

Figura 1 - Blocos para análise das tensões no arco de contato

Para se deduzir a equação de equilíbrio no ponto de entrada $\mathrm{B}$ deve-se isolar o bloco $\mathrm{B}$, de espessura infinitesimal e lados perpendiculares ao plano de laminação, caracterizado pelo ângulo $\varphi$, conforme mostrado na figura 2 a seguir.

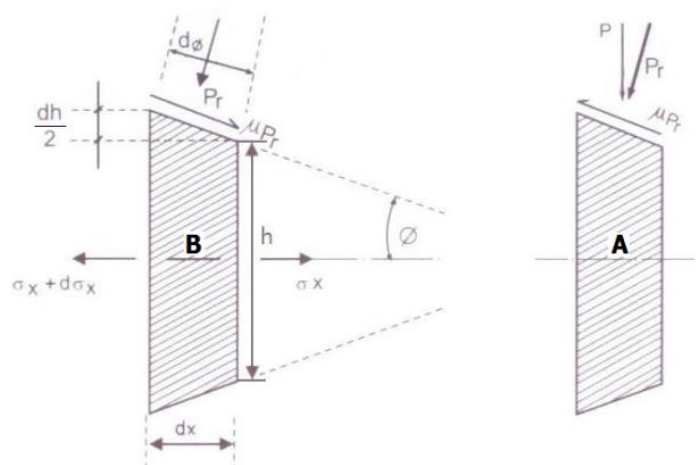

Figura 2 - Tensões nos blocos infinitesimais isolados

Analisando-se o bloco $\mathrm{B}$, pode-se facilmente chegar à expressão

$$
\frac{d h}{2}=d x \tan \varphi=R^{\prime} d \varphi \sin \varphi
$$

Bland \& Ford (apud Helman et al. [2]) consideram que a largura da chapa é unitária e estudam as componentes de todas as forças que atuam em cada bloco separadamente. Adotando-se como positivas as forças nos blocos que se dirigem da direita para a esquerda, obtêm-se as seguintes forças na direção $x$ :

$i$ - Devido às tensões longitudinais:

$$
\left(\sigma_{x}+d \sigma_{x}\right)(h+d h)-h \sigma_{x}
$$

$i i$ - Devido à pressão radial $\left(P_{r}\right)$ de ambos os cilindros na direção $x$ :

* Contribuição técnica ao 69 Congresso Anual da ABM - Internacional e ao 14ํㅡㄹ ENET - Encontro Nacional de Estudantes de Engenharia Metalúrgica, de Materiais e de Minas, 21 a 25 de julho de 2014, São Paulo, SP, Brasil. 


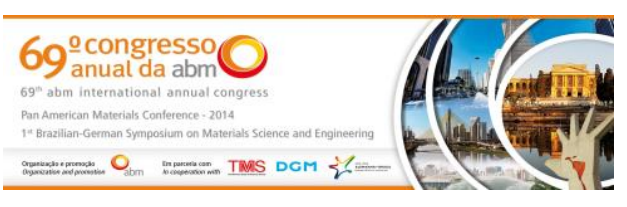

$$
2\left(P_{r} \frac{d x}{\cos \varphi}\right) \sin \varphi
$$

$i i i$ - Devido às forças de atrito $\left(\mu P_{r}\right)$ nos dois cilindros na direção $x$ :

$$
2 \mu\left(P_{r} \frac{d x}{\cos \varphi}\right) \cos \varphi
$$

Aplicando-se a condição de equilíbrio $\left(\sum F_{x}=0\right)$ nos dois blocos separadamente, pode-se reunir a análise em uma única expressão dada por

$$
\sigma_{x} d h+h d \sigma_{x}+2 P_{r} d x \tan \varphi \pm 2 \mu P_{r} d x=0
$$

Bland \& Ford (apud Helman et al. [2]) desenvolveram essa condição de equilíbrio para os dois blocos e chegaram às expressões que definem a distribuição de pressão nas regiões de saída e entrada do arco de contato após algumas simplificações e considerável trabalho algébrico.

A distribuição de pressão nas regiões de saída e entrada do arco de contato, sem a aplicação de tensões externas, podem ser dadas respectivamente pelas expressões:

$$
\frac{P^{+}}{S}=\frac{h}{h_{f}} \exp (+\mu \cdot H(\varphi)) \quad \text { e } \quad \frac{P^{-}}{S}=\frac{h}{h_{i}} \exp \left[\mu\left(H_{i}(\varphi)-H(\varphi)\right)\right]
$$

Onde $H(\varphi)$ é dado por

$$
H(\varphi)=2 \sqrt{\frac{R^{\prime}}{h_{f}}} \operatorname{arctg}\left(\sqrt{\frac{R^{\prime}}{h_{f}}} \varphi\right)
$$

As mesmas expressões podem ser definidas para a situação em que se tem a aplicação de tensões externas, sendo dadas por

$$
\frac{P^{+}}{S}=\left(1-\frac{t_{f}}{S_{f}}\right) \frac{h}{h_{f}} \exp (+\mu . H(\varphi)) \quad \text { e } \quad \frac{P^{-}}{S}=\left(1-\frac{t_{i}}{s_{i}}\right) \frac{h}{h_{i}} \exp \left[\mu\left(H_{i}(\varphi)-H(\varphi)\right)\right]
$$

Pode-se calcular a posição do ângulo neutro $\alpha_{N}$ fazendo-se $P^{+}=P^{-}$nas equações anteriores, de onde se obtém para o ângulo neutro a expressão:

$$
\alpha_{N}=\sqrt{\frac{h_{f}}{R^{\prime}}} \operatorname{tg}\left[\frac{H_{N}}{2}\left(\sqrt{\frac{h_{f}}{R^{\prime}}}\right)\right]
$$

Onde $H_{N}$ é dado por

$$
H_{N}=\frac{H_{i}}{2}-\frac{1}{2 \cdot \mu} \ln \left[\frac{h_{i}\left(1-\frac{t_{f}}{S_{f}}\right)}{h_{f}\left(1-\frac{t_{i}}{S_{i}}\right)}\right]
$$

Bland \& Ford (apud Helman et al. [2]) afirmam que pode-se calcular a carga de laminação pela integral da função distribuição de pressão nas regiões de saída (+) e entrada (-) do arco de contato dos cilindros de laminação.

* Contribuição técnica ao 69 Congresso Anual da ABM - Internacional e ao 14ํㅡㄹ ENET - Encontro Nacional de Estudantes de Engenharia Metalúrgica, de Materiais e de Minas, 21 a 25 de julho de 2014, São Paulo, SP, Brasil. 


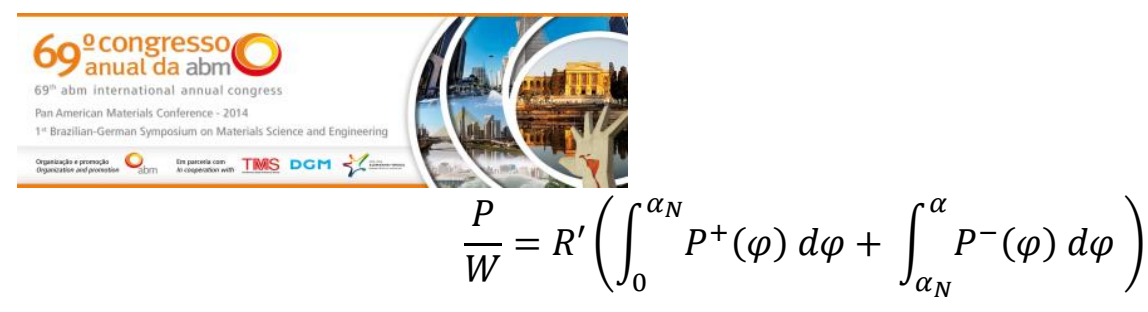

As expressões que definem a distribuição de pressão no arco de contato podem ser representadas graficamente pela "colina de fricção", da figura 3.

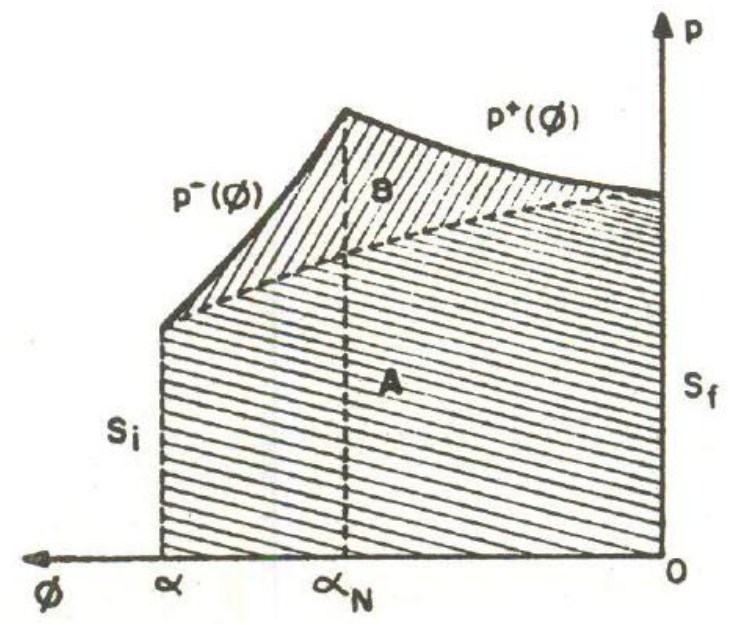

Figura 3 - Colina de fricção da teoria da laminação

Nela pode-se visualizar duas regiões: a região $A$, que representa a força necessária à deformação homogênea do material sem atrito, e a região $B$, que representa a força adicional necessária devido ao atrito. Pode-se usar o critério de escoamento de Tresca (apud Plaut [4]) para se calcular a tensão longitudinal $\sigma_{x}$ em um ponto qualquer do arco de contato.

\section{MATERIAIS E MÉTODOS}

O experimento foi realizado laminando-se barras chatas extrudadas da liga de Alumínio 6063-T5 no laminador Machine duo reversível de 8 toneladas. As amostras foram cortadas com $300 \mathrm{~mm}$ de comprimento a partir de um perfil retangular possuindo valores nominais de $50,8 \mathrm{~mm}\left(2^{\prime}\right)$ de largura por $6,35 \mathrm{~mm}$ $\left(1 / 4^{\prime}\right)$ de espessura, conforme figura 4 a seguir.

* Contribuição técnica ao 69 Congresso Anual da ABM - Internacional e ao 14ํㅡㄹ ENET - Encontro Nacional de Estudantes de Engenharia Metalúrgica, de Materiais e de Minas, 21 a 25 de julho de 2014, São Paulo, SP, Brasil. 


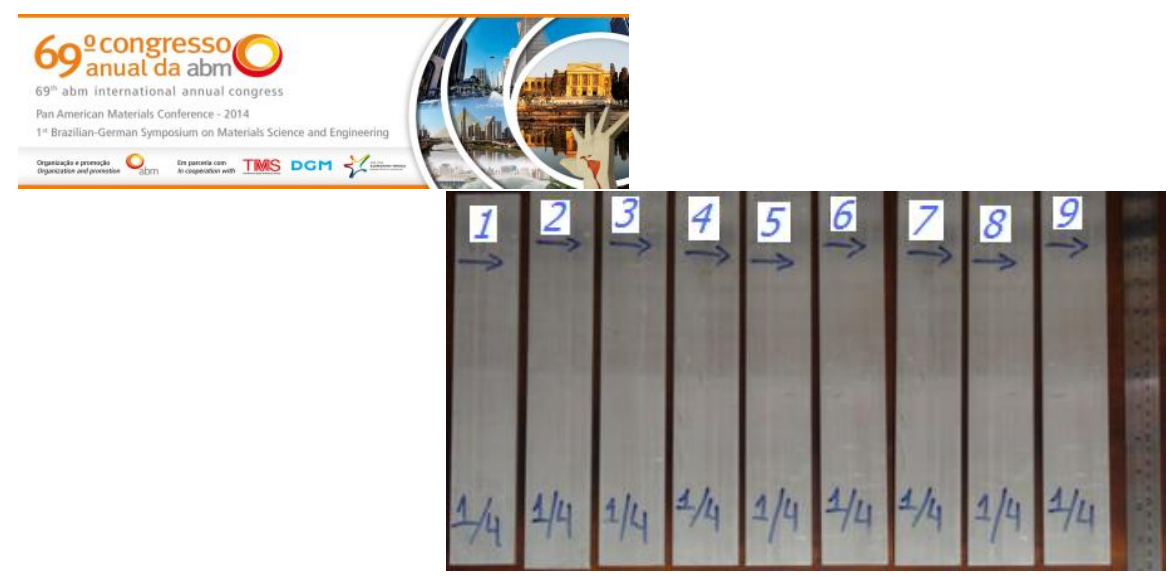

Figura 4 - Amostras da liga de Alumínio 6063-T5

O fabricante [5] atesta que as propriedades mecânicas das barras são conforme NBR 7000 (Propriedades mecânicas do produto), com Limite de Escoamento Mínimo (LE min) de 105 Mpa, Limite de Resistência Mínimo (LRT min) de 145 Mpa, Alongamento Mínimo de 8\% e Dureza Brinell de 66 HB, e a composição química conforme NBR 6834 (Alumínio e suas ligas).

Tabela 1 - Composição química (wt\%) da liga de Alumínio 6063-T5

\begin{tabular}{cccccccccc}
\hline Al & $\mathrm{Si}$ & $\mathrm{Fe}$ & $\mathrm{Cu}$ & $\mathrm{Mn}$ & $\mathrm{Mg}$ & $\mathrm{Cr}$ & $\mathrm{Zn}$ & $\mathrm{Ti}$ & Outros \\
\hline Restante & $0,20-$ & 0,35 & 0,10 & 0,10 & $0,45-0,90$ & 0,10 & 0,10 & 0,10 & 0,15 \\
& 0,60 & & & & & & & & \\
\hline
\end{tabular}

Os equipamentos e materiais utilizados no experimento foram:

a) Laminador Machine duo reversível de 8 toneladas;

b) Nove barras chatas extrudadas de $\left(1 / 4^{\prime}\right)$ de espessura;

c) Escala de medição de $600 \mathrm{~mm}$, com resolução de $1 \mathrm{~mm}$;

d) Paquímetro universal, com resolução de 0,05 $\mathrm{mm}$.

O laminador Machine possui dois cilindros confeccionados em aço AISI 52100 com diâmetro de $55 \mathrm{~mm}$ e dureza superficial de $60 \mathrm{RC}$. Segundo Thomas [6], a composição química do aço AISI 52100 é conforme a seguir.

Tabela 2- Composição química (wt\%) do aço AISI 52100

\begin{tabular}{ccccccc}
\hline $\mathrm{Fe}$ & $\mathrm{Cr}$ & $\mathrm{C}$ & $\mathrm{Mn}$ & $\mathrm{Si}$ & $\mathrm{S}$ & $\mathrm{P}$ \\
\hline $96,5-$ & $1,30-1,60$ & $0,98-$ & $0,25-$ & $0,15-$ & $<0,025$ & $<0,025$ \\
97,32 & & 1,10 & 0,45 & 0,30 & & \\
\hline
\end{tabular}

Algumas propriedades físicas e mecânicas associadas ao material dos cilindros do laminador são essenciais aos modelos matemático computacionais usados na laminação de tiras a frio, como o Módulo de Elasticidade e o Coeficiente de Poisson do aço dos cilindros do laminador.

Tabela 3 - Algumas propriedades do aço AISI 52100

\begin{tabular}{lc}
\hline Propriedades & Valores \\
\hline Densidade & $7,81 \mathrm{~g} / \mathrm{cm}^{3}$ \\
\hline Ponto de Fusão & $1424^{\circ} \mathrm{C}$ \\
\hline Módulo de Elasticidade & $190-210 \mathrm{GPa}$ \\
\hline Coeficiente de Poisson & $0,27-0,30$ \\
\hline
\end{tabular}

O procedimento adotado na realização do experimento consistiu-se inicialmente na tomada das dimensões de nove (9) amostras em liga de Alumínio 6063-T5, seguindo-se sua laminação. Após isso, foram novamente tomadas as dimensões das amostras objetivando o registro do grau de deformação imposto sobre cada

* Contribuição técnica ao 69ํ Congresso Anual da ABM - Internacional e ao 14ํㅡN Nacional de Estudantes de Engenharia Metalúrgica, de Materiais e de Minas, 21 a 25 de julho de 2014, São Paulo, SP, Brasil. 


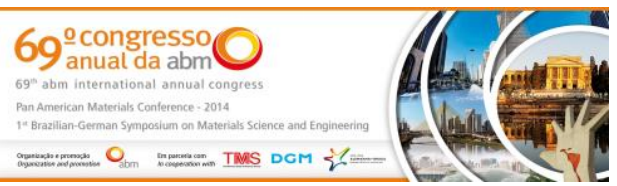

uma das amostras selecionadas. Posteriormente realizou-se a implementação dos modelos matemáticos descritos via rotinas computacionais, as quais foram desenvolvidas específicas para esse fim. A figura 5 resume o procedimento adotado.

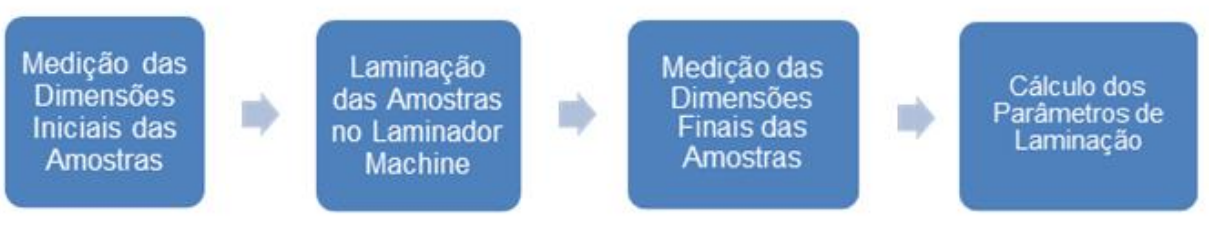

Figura 5 - Diagrama do procedimento experimental realizado no laminador

\section{RESULTADOS E DISCUSSÃO}

Os dados foram obtidos pelo uso manual da escala de medição e paquímetro universal analógico, justificando-se os valores apresentados com resoluções diferenciadas. Os valores medidos para as barras chatas de $\left(1 / 4^{\prime}\right)$ de espessura foram tomados para abertura (gap) decrescente dos cilindros do laminador, ou seja, a última amostra foi aquela que sofreu maior deformação devido à menor espessura da abertura entre os cilindros.

Tabela 4 - Dados das amostras de barras chatas 6063-T5 de 1/4

\begin{tabular}{lccccccc}
\hline Amostras & $\mathrm{l}_{\mathrm{i}}$ & $\mathrm{W}_{\mathrm{i}}$ & $\mathrm{h}_{\mathrm{i}}$ & gap & $\mathrm{l}_{\mathrm{f}}$ & $\mathrm{W}_{\mathrm{f}}$ & $\mathrm{h}_{\mathrm{f}}$ \\
\hline Amostra 1 & 292,5 & 50,70 & 6,10 & 6,20 & 292,7 & 50,70 & 6,10 \\
\hline Amostra 2 & 294,6 & 50,80 & 6,10 & 6,00 & 295,0 & 50,80 & 6,05 \\
\hline Amostra 3 & 300,6 & 50,80 & 6,10 & 5,80 & 302,8 & 50,80 & 6,00 \\
\hline Amostra 4 & 301,8 & 50,80 & 6,10 & 5,60 & 309,5 & 50,80 & 5,95 \\
\hline Amostra 5 & 301,6 & 50,80 & 6,10 & 5,40 & 318,5 & 50,80 & 5,70 \\
\hline Amostra 6 & 301,4 & 50,80 & 6,10 & 5,20 & 332,5 & 50,90 & 5,50 \\
\hline Amostra 7 & 300,2 & 50,80 & 6,10 & 5,00 & 341,0 & 50,90 & 5,30 \\
\hline Amostra 8 & 300,9 & 50,80 & 6,10 & 4,80 & 356,0 & 51,00 & 5,10 \\
\hline Amostra 9 & 301,5 & 50,80 & 6,10 & 4,70 & 361,0 & 51,00 & 5,00 \\
\hline
\end{tabular}

Finalizada a coleta de dados das amostras laminadas iniciou-se a fase de análise e processamento dos mesmos a partir de rotinas de cálculo computacionais desenvolvidas para esse fim. Os valores das cargas de laminação estimados pelos modelos de Orowan, Ekelund e Bland \& Ford, implementados para os dados obtidos são apresentados a seguir.

Tabela 5 - Valores estimados para a carga total de laminação (t)

\begin{tabular}{lccccccccc}
\hline Discretização & 1 & 2 & 3 & 4 & 5 & 6 & 7 & 8 & 9 \\
\hline Orowan & 0 & 0,8844 & 1,2507 & 1,5318 & 2,5013 & 3,0665 & 3,5409 & 3,9628 & 4,1562 \\
\hline Ekelund & 0 & 0,7877 & 1,1098 & 1,3588 & 2,2202 & 2,7161 & 3,1234 & 3,4754 & 3,6326 \\
\hline Bland \& Ford & 0 & 0,4518 & 0,7025 & 0,9140 & 1,7530 & 2,3055 & 2,8030 & 3,2608 & 3,4769 \\
\hline
\end{tabular}

Pode-se observar que o modelo de Orowan estimou os maiores valores para a carga de laminação e o modelo de Bland \& Ford os menores. Os valores estimados por Ekelund se aproximaram daqueles do modelo de Bland \& Ford para as amostras que sofreram as maiores deformações e do modelo de Orowan para as amostras com as menores deformações.

* Contribuição técnica ao 69 Congresso Anual da ABM - Internacional e ao 14ํㅡㄹ ENET - Encontro Nacional de Estudantes de Engenharia Metalúrgica, de Materiais e de Minas, 21 a 25 de julho de 2014, São Paulo, SP, Brasil. 

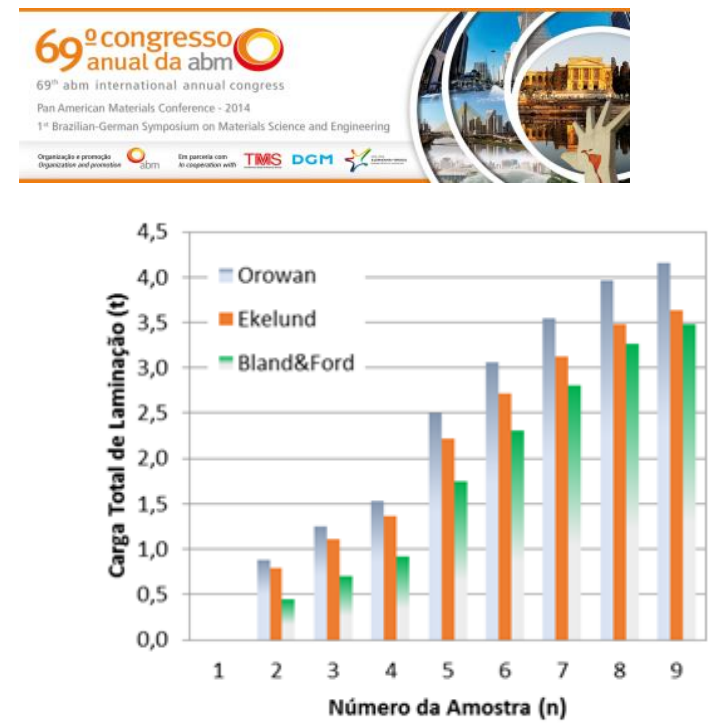

(a)

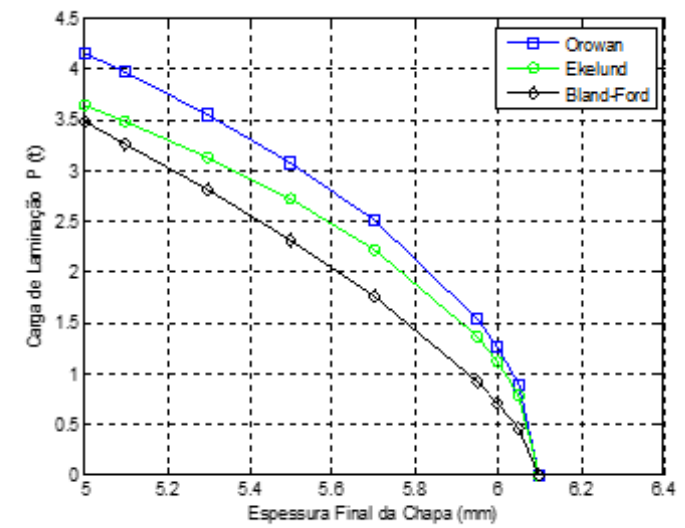

(b)

Figura 6. (a) e (b) - Comparativo entre modelos de predição da carga.

A figura 6 (b) mostra a variação da carga total de laminação estimada pelos modelos de Orowan, Ekelund e Bland \& Ford como função da espessura final da chapa laminada. Trata-se da curva carga-deformação da chapa.

Foi implementado uma rotina computacional específica para se analisar os dados segundo o modelo de Bland \& Ford. Essa rotina gera os valores de pressão específica ao longo do arco de contato para cada amostra e realiza em seguida a integração desses valores para se obter a carga total nos cilindros de laminação. Os valores de carga total gerados para cada amostra foram apresentados na tabela 5. Além dos valores da carga de laminação, a rotina Bland \& Ford permite visualizar a variação da espessura da chapa, a deformação e a colina de fricção ao longo do arco de contato para cada amostra ensaiada. A figura 7 a seguir apresenta a variação da espessura 7(a) e da deformação 7(b) para as amostras 2, 6 e 9 respectivamente.

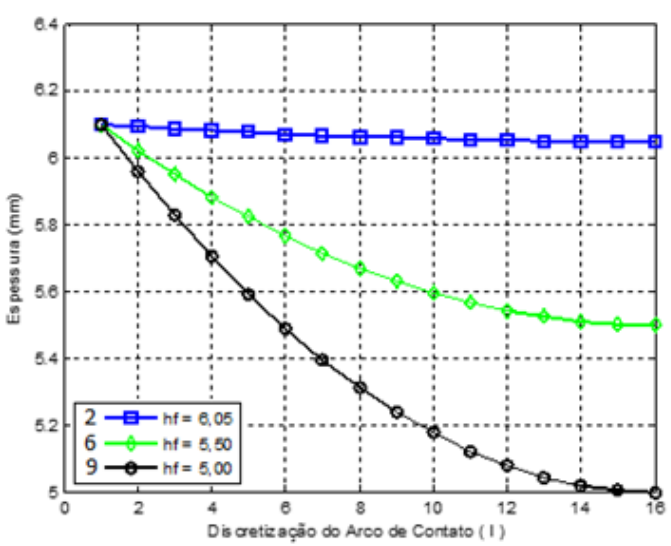

(a)

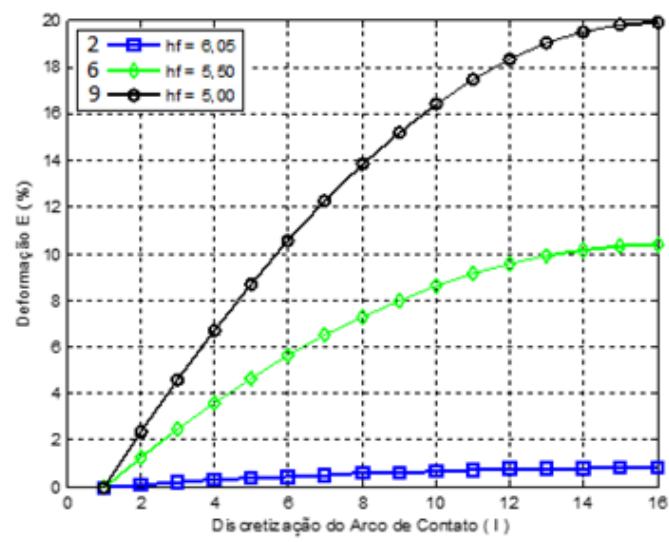

(b)

Figura 7. (a) e (b) - Espessura e deformação no arco de contato.

Pode-se observar que à medida que se reduz a espessura final da amostra laminada aumenta-se a deformação sobre a mesma ao longo do arco de contato, atingindo-se um valor final acumulado correspondente à espessura final da chapa. As colinas de fricção obtidas para as amostras 2, 6 e 9 são apresentadas nas figuras a seguir. A distinção entre as regiões de entrada e saída no arco de contato pode ser visualizada pelo valor de pico da distribuição de pressão.

* Contribuição técnica ao 69 Congresso Anual da ABM - Internacional e ao 14ํㅡㄹ ENET - Encontro Nacional de Estudantes de Engenharia Metalúrgica, de Materiais e de Minas, 21 a 25 de julho de 2014, São Paulo, SP, Brasil. 


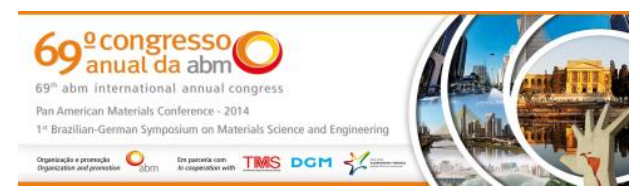

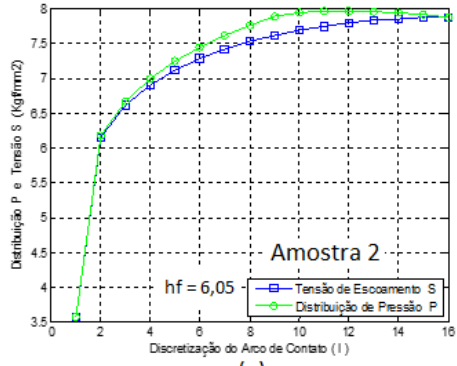

(a)

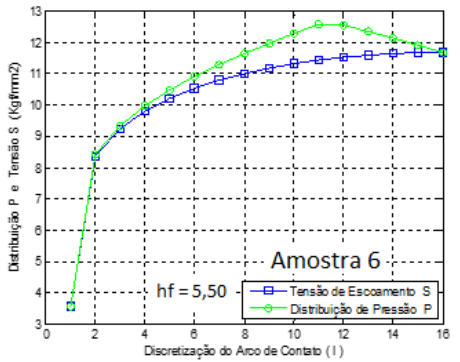

(b)

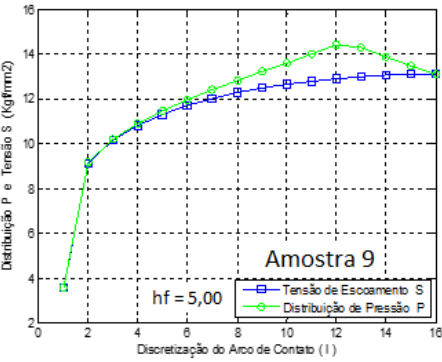

(c)

Figura 8. (a), (b) e (c) - Colina de fricção para as amostras 2, 6 e 9

Como se pode observar, o material apresenta maior resistência à deformação para produzir as menores espessuras finais, o que implica naturalmente em maiores valores para a carga de laminação, atestados pela tensão de escoamento $\mathrm{S}$ e pressão específica $\mathrm{P}$ crescentes para as amostras citadas.

\section{CONCLUSÃO}

a) Os modelos de Orowan, Ekelund e Bland \& Ford possibilitaram a predição e a análise das cargas de laminação envolvidas no processo de laminação a partir de sua implementação por rotinas de cálculo computacionais. A definição de qual modelo melhor se aproxima dos valores reais da carga de laminação nos cilindros do laminador será possível apenas após a instalação do sistema de medição força no mesmo.

b) As rotinas de cálculo mostram-se como boas ferramentas para análise não somente da distribuição de pressão $P$ e tensão de escoamento $S$ ao longo do arco de contato mas também podem ser usadas na análise de outras grandezas importantes na laminação, tais como a velocidade de saída da chapa e ângulo neutro no arco de contato. Tais rotinas apresentam potencial para análise de problemas de laminação em linhas de produção industriais bem como no projeto de novas linhas.

c) O uso de rotinas de processamento matemático computacional tornam-se fundamentais para a análise dos dados coletados nos processos e implementação de quaisquer modelos matemáticos para a determinação não somente da carga de laminação como de outros parâmetros importantes.

\section{Agradecimentos}

Agradecimento ao IFES - Instituto Federal do Espírito Santo, que disponibilizou o laminador Machine duo reversível para a realização dos experimentos, ao meu orientador, Prof. Dr. Marcelo Lucas Pereira Machado e em especial ao Prof. Dr. José Denti Filho, pelo apoio prestado na elaboração desse trabalho.

\section{REFERÊNCIAS}

1 Machado MLP. Conformação dos Metais: Fundamentos e Aplicações. Vitória: Instituto Federal do Espírito Santo, 2009.

2 Helman H, et al. Fundamentos da Laminação: Produtos Planos. São Paulo: ABM, 1988.

3 Ginzburg VB. Steel-Rolling Technology: Theory and Practice. New York: Marcel Dekker, 1989.

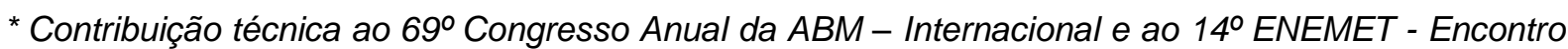
Nacional de Estudantes de Engenharia Metalúrgica, de Materiais e de Minas, 21 a 25 de julho de 2014, São Paulo, SP, Brasil. 
4 Plaut RL. Laminação de Aços: Tópicos Avançados. 3. ed. São Paulo: ABM, 1987.

5 Alumiplast Comércio de Metais LTDA. Composição Química - Extrudados. Alumiplast Produtos em Alumínio: Indústria e Construção Civil. Disponivel em: <http://www.alumiplast.com.br/documentos/composicao_quimica.pdf>. Acesso em: 29 maio 2013.

6 Thomas G. AISI 52100 Alloy Steel (UNS G52986). AZoM.com: the A to Z of Materials, 2012. Disponivel em: <http://www.azom.com/article.aspx?ArticlelD=6704>. Acesso em: 5 Julho 2013.

\section{BIBLIOGRAFIA}

1 Helman H, Cetlin PR. Fundamentos da Conformação Mecânica dos Metais. 2. ed. São Paulo: Artliber, 2005.

2 Schaeffer L. Conformação Mecânica. Porto Alegre: Imprensa Livre, 1999.

3 Callister WD. Fundamentos da Ciência e Engenharia de Materiais: Uma Abordagem Integrada. 2a. ed. Rio de Janeiro: LTC - Livros Técnicos e Científicos, 2006.

4 Machado MLP. Modelo Termomicroestrutural para Determinação de Variáveis Operacionais e Metalúrgicas para Utilização em Simulação e/ou Controle na Laminação a Quente de Aços Carbono e Microligados. Tese de Doutorado. PPGEE-UFES. Vitória-ES. 2005.

5 Denti JF. Um Método de Controle Dinâmico de Laminadores Reversíveis. Tese de Doutorado. PPGEM-EEUFMG. Belo Horizonte-MG. 1994.

6 Associação Brasileira de Metais ABM. Laminação dos Aços. 4. ed. São Paulo: [s.n.], 1981.

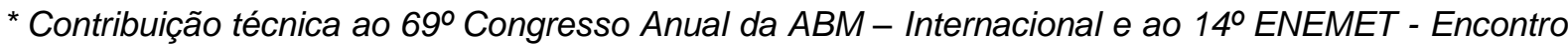
Nacional de Estudantes de Engenharia Metalúrgica, de Materiais e de Minas, 21 a 25 de julho de 2014, São Paulo, SP, Brasil. 